\title{
ALMA unveils rings and gaps in the protoplanetary system HD 169142: signatures of two giant protoplanets
}

\author{
D. Fedele ${ }^{1}$, M. Carney ${ }^{2}$, M. R. Hogerheijde ${ }^{2}$, C. Walsh ${ }^{2,3}$, A. Miotello ${ }^{2}$, P. Klaassen ${ }^{4}$, S. Bruderer $^{5}$, \\ Th. Henning ${ }^{6}$, and E. F. van Dishoeck ${ }^{2,5}$
}

\author{
1 INAF-Osservatorio Astrofisico di Arcetri, L.go E. Fermi 5, 50125 Firenze, Italy \\ e-mail: fedele@arcetri.astro.it \\ 2 Leiden Observatory, Leiden University, PO Box 9513, 2300 RA, Leiden, The Netherlands \\ 3 School of Physics and Astronomy, University of Leeds, Leeds LS2 9JT, UK \\ ${ }^{4}$ UK Astronomy Technology Centre, Royal Observatory Edinburgh, Blackford Hill, Edinburgh EH9 3HJ, UK \\ 5 Max Planck Institut für Extraterrestrische Physik, Giessenbachstrasse 1, 85748 Garching, Germany \\ ${ }^{6}$ Max-Planck-Institute for Astronomy, Koenigstuhl 17, 69117 Heidelberg, Germany
}

Received 7 October 2016 / Accepted 8 February 2017

\begin{abstract}
The protoplanetary system HD 169142 is one of the few cases where a potential candidate protoplanet has recently been detected by direct imaging in the near-infrared. To study the interaction between the protoplanet and the disk itself, observations of the gas and dust surface density structure are needed. This paper reports new ALMA observations of the dust continuum at $1.3 \mathrm{~mm},{ }^{12} \mathrm{CO},{ }^{13} \mathrm{CO}$, and $\mathrm{C}^{18} \mathrm{O} J=2-1$ emission from the system HD 169142 (which is observed almost face-on) at an angular resolution of $\sim 0^{\prime \prime} 3 \times 0 \times 0^{\prime \prime} \cdot 2$ $(\sim 35 \times 20 \mathrm{au})$. The dust continuum emission reveals a double-ring structure with an inner ring between $00^{\prime \prime} 17-00^{\prime \prime} 28(\sim 20-35$ au $)$ and an outer ring between $0{ }^{\prime \prime} 48-00^{\prime \prime} 64(\sim 56-83 \mathrm{au})$. The size and position of the inner ring is in good agreement with previous polarimetric observations in the near-infrared and is consistent with dust trapping by a massive planet. No dust emission is detected inside the inner dust cavity ( $R \lesssim 20 \mathrm{au}$ ) or within the dust gap ( $\sim 35-56 \mathrm{au})$ down to the noise level. In contrast, the channel maps of the $J=2-1$ line of the three $\mathrm{CO}$ isotopologs reveal gas inside the dust cavity and dust gap. The gaseous disk is also much larger than the compact dust emission; it extends to $\sim 1^{\prime \prime} .5(\sim 180 \mathrm{au})$ in radius. This difference and the sharp drop of the continuum emission at large radii point to radial drift of large dust grains ( $>\mu \mathrm{m}$ size). Using the thermo-chemical disk code DALI, we modeled the continuum and the $\mathrm{CO}$ isotopolog emission to quantitatively measure the gas and dust surface densities. The resulting gas surface density is reduced by a factor of $\sim 30-40$ inward of the dust gap. The gas and dust distribution indicate that two giant planets shape the disk structure through dynamical clearing (dust cavity and gap) and dust trapping (double-ring dust distribution).
\end{abstract}

Key words. stars: variables: T Tauri, Herbig Ae/Be - protoplanetary disks - planet-disk interactions

\section{Introduction}

Observations of the cold gas and dust reservoirs of protoplanetary disks are powerful tools for investigating the early phase of planet formation. In particular, addressing the radial distribution of gas and dust in the disk interior has the potential to unveil the initial conditions for the formation of gas giant planets. In contrast with optical and near-infrared scattered light data or infrared spectroscopy, submillimeter observations probe the bulk of the gas and dust mass in the disk. Recent observations in the submillimeter regime with the Atacama Large Millimetre/Submillimeter Array (ALMA) brought to light evidence of discontinuous radial (gaps and cavities) and azimuthal (traps and vortices) dust distributions (e.g., Casassus et al. 2012; van der Marel et al. 2013; ALMA Partnership et al. 2015; Walsh et al. 2014; Andrews et al. 2016; Isella et al. 2016) and cold gas cavities that are smaller than the dust cavities (van der Marel et al. 2016).

Different theories have been proposed to explain the formation of dust gaps and rings, including dynamical interaction with one or more giant planets that carve out the dust (e.g., Papaloizou \& Lin 1984), magneto-rotational instability that creates dead-zones (Flock et al. 2015), dust grain growth corresponding to condensation fronts (Zhang et al. 2015), fusing of dust grains at temperatures below the sublimation point (dust sintering, Okuzumi et al. 2016), and photoevaporation (Ercolano et al. 2017).

Submillimeter interferometric observations of both gas and dust may help to unveil the evolutionary status of protoplanetary systems and to distinguish the physical origin of dust cavities and dust gaps in disks. A particularly powerful test is to perform ALMA observations of disks for which optical and near-infrared data have previously suggested embedded planets.

\section{HD 169142}

HD 169142 is a young $6_{-3}^{+6} \mathrm{Myr}$ and isolated (Grady et al. 2007) Herbig Ae/Be star (The et al. 1994) with $M_{\star}=1.65 M_{\odot}$ (Blondel \& Djie 2006), spectral type A5, and $T_{\text {eff }}=8400 \mathrm{~K}$ (Dunkin et al. 1997). The most recent measurement of the parallax is $\varpi=8.53 \pm 0.29$ mas (Gaia Collaboration 2016), which translates into a distance $d=117 \pm 4 \mathrm{pc}$. Previous estimates by de Zeeuw et al. (1999) instead were $d=145 \mathrm{pc}$. In the remaining paper we use the newest distance estimate and adjust all the relevant parameters. The stellar luminosity adopted in this paper is $L_{\star}=10 L_{\odot}$ based on the new distance estimate and on the optical $V$-magnitude and extinction $(V=8.15 \mathrm{mag}$, $A_{\mathrm{V}}=0.43$ mag, e.g., Malfait et al. 1998). Submillimeter observations of the $1.3 \mathrm{~mm}$ dust continuum and $\mathrm{CO} J=2-1$ with the Submillimeter Array (SMA) measured a disk inclination of $13^{\circ}$ and a position angle of $5^{\circ}$ (Raman et al. 2006; Panić et al. 2008). 
Table 1. HD 169142 observational parameters.

\begin{tabular}{|c|c|c|c|}
\hline \multirow[t]{2}{*}{$\begin{array}{l}\text { Dates observed } \\
\text { baselines }\end{array}$} & \multicolumn{3}{|c|}{$\begin{array}{c}2015 \text { August } 30 \\
13-1445 \mathrm{~m} \mid 10-1120 \mathrm{k} \lambda\end{array}$} \\
\hline & $\mathrm{C}^{18} \mathrm{O} 2-1$ & ${ }^{13} \mathrm{CO} 2-1$ & ${ }^{12} \mathrm{CO} 2-1$ \\
\hline Rest frequency [GHz] & 219.56035 & 220.39868 & 230.53800 \\
\hline Synthesized beam $[F W H M]$ & $0 \prime: 36 \times 0,23$ & $0 \prime 37 \times 0,: 22$ & $0^{\prime \prime} 37 \times 00^{\prime \prime}: 20$ \\
\hline Position angle & -74.5 & $-75: 2$ & $-72: 8$ \\
\hline Channel width $\left[\mathrm{km} \mathrm{s}^{-1}\right]$ & 0.084 & 0.084 & 0.040 \\
\hline rms noise (per channel) $\left[\mathrm{mJy}_{\text {beam }}{ }^{-1}\right]$ & 6 & 8 & 13 \\
\hline Peak emission $\left[\mathrm{mJy} \mathrm{beam}^{-1}\right]$ & 100 & 200 & 540 \\
\hline $\begin{array}{l}\text { Integrated flux }{ }^{a}\left[\mathrm{Jy} \mathrm{km} \mathrm{s}^{-1}\right] \\
\text { Weighting }\end{array}$ & $3.9 \pm 0.5$ & $\begin{array}{l}7.6 \pm 0.6 \\
\text { natural }\end{array}$ & $14.0 \pm 2.0$ \\
\hline Continuum frequency $[\mathrm{GHz}]$ & & 233.0 & \\
\hline Synthesized beam $[F W H M]$ & $0 \prime 28 \times 0 \prime \prime 18$ & $0 \prime 224 \times 0,: 16$ & $0 \prime 22 \times 00^{\prime \prime} 14$ \\
\hline Position angle & $-77 \div 9$ & $-78: 3$ & $-80: 0$ \\
\hline rms noise $\left[\mathrm{mJy} \mathrm{beam}^{-1}\right]$ & 0.07 & 0.26 & 0.11 \\
\hline Peak emission $\left[\mathrm{mJy}_{\text {beam }}{ }^{-1}\right]$ & 17 & 15 & 13 \\
\hline Integrated flux [mJy] & $232 \pm 23$ & $226 \pm 23$ & $226 \pm 23$ \\
\hline Weighting & Briggs, robust $=0.5$ & Uniform & Superuniform \\
\hline
\end{tabular}

Notes. Flux calibration accuracy is taken to be $10 \%$. ${ }^{(a)}$ Integrated over a circular aperture of 3 " radius.

Based on observations of multiple $\mathrm{CO}$ isotopologs with the SMA, Panić et al. (2008) derived a total gas mass of 0.6-3.0 $\times$ $10^{-2} M_{\odot}$ in good agreement with the estimate by Pinte et al. (2010) of $\sim 10^{-2} M_{\odot}$ based on the Herschel/PACS detection of [O I] $63 \mu \mathrm{m}$ (Meeus et al. 2010; Fedele et al. 2013). The spectral energy distribution (SED) suggests a discontinuous radial distribution of the dust (Malfait et al. 1998). This is confirmed by direct imaging observations of the thermal emission at mid-infrared wavelengths (Honda et al. 2012) as well as by $H$-band scattered light emission (Fukagawa et al. 2010; Quanz et al. 2013; Momose et al. 2015; Wagner et al. 2015). In particular, the $H$-band polarimetric image shows a ring-like dust distribution at a radius of $\sim 0 \prime \prime 17$ (20 au) from the star. The dust ring seen in scattered light is also detected in the $7 \mathrm{~mm}$ continuum by Osorio et al. (2014). The latter find evidence of a second dust gap beyond the inner ring.

Biller et al. (2014) and Reggiani et al. (2014) detected a point-like emission at $\sim 0$ '.11-0'.16 (13-18 au) in $L^{\prime}$-band coronographic and polarimetric differential imaging, respectively. According to Reggiani et al. (2014), this emission is produced by a massive planet of $\sim 30 M_{\text {Jupiter }}$. Biller et al. (2014) instead warned that because of the non-detection in the $H$ and $K_{\mathrm{s}}$ bands, this might be a disk feature. Nevertheless, the potential discovery of a protoplanet makes HD 169142 an ideal case to study the planet-disk interaction during the early phases of planetary formation and evolution.

This paper presents new ALMA high angular resolution observations of HD 169142 of the $J=2-1$ transition of ${ }^{12} \mathrm{CO}$, ${ }^{13} \mathrm{CO}$, and $\mathrm{C}^{18} \mathrm{O}$ and $1.3 \mathrm{~mm}$ dust continuum. From these observations we place more stringent constraints on the dust and gas density structures. In Sect. 3 we summarize the observing strategy and the data reduction. Results are presented in Sect. 4. In Sect. 5 we compare the observations with simulations of thermochemical disk models to constrain the gas and dust distributions. Discussion and conclusions are given in Sects. 6 and 7, respectively.

\section{Observations and data reduction}

HD $169142\left(\mathrm{~J} 2000:\right.$ RA $=18^{\mathrm{h}} 24^{\mathrm{m}} 29.776^{\mathrm{s}}$, Dec $=$ $\left.-29^{\circ} 46^{\prime} 49.900^{\prime \prime}\right)$ was observed on 2015 August 30 with ALMA in band $6(211-275 \mathrm{GHz})$ as part of project 2013.1.00592.S. In total, 35 antennas were used to achieve a spatial resolution of $\sim 0 \prime$ '.2-0'.3. The upper sideband (USB) contained two spectral windows. One window has continuum observations in the time domain mode (TDM) correlator setting with $2 \mathrm{GHz}$ bandwidth centered at $233 \mathrm{GHz}$. The ${ }^{12} \mathrm{CO} J=2-1$ line at $230.538 \mathrm{GHz}$ was observed in the second USB spectral window with the frequency domain mode (FDM) correlator setting at $30.5 \mathrm{kHz}$ $\left(0.040 \mathrm{~km} \mathrm{~s}^{-1}\right)$ frequency (velocity) resolution. ${ }^{13} \mathrm{CO} J=2-1$ at $220.39868 \mathrm{GHz}$ and $\mathrm{C}^{18} \mathrm{O} J=2-1$ at $219.56035 \mathrm{GHz}$ were both observed in separate spectral windows in the lower sideband (LSB) in FDM mode. Each observed LSB line had a frequency (velocity) resolution of $61.0 \mathrm{kHz}\left(0.084 \mathrm{~km} \mathrm{~s}^{-1}\right)$. Table 1 summarizes the observational parameters.

Visibility data were obtained in a single execution block with a $6.05 \mathrm{~s}$ integration time per visibility for $50 \mathrm{~min}$ total on-source. System temperatures were between 50-200 K. Weather conditions on the date of observation gave an average precipitable water vapor of $1.8 \mathrm{~mm}$. Calibration was made with J1924-2914 as the delay and bandpass calibrator, J1812-2836 as the gain calibrator, and Ceres as the flux calibrator. The flux values for Ceres on the date of observation were $1.941 \mathrm{Jy}$ in the LSB and $2.165 \mathrm{Jy}$ in the USB. The visibility data were subsequently time binned to $30 \mathrm{~s}$ integration times per visibility for self-calibration, imaging, and analysis. Extended emission is present in ${ }^{12} \mathrm{CO}$ and ${ }^{13} \mathrm{CO}$ data and is poorly sampled on short baselines in the $u v$ space, which resulted in a reduced image quality. For this reason, the total flux listed in Table 1 is integrated in a circular aperture of $3^{\prime \prime}$ radius centered on the source position.

Self-calibration for HD 169142 was performed using the $233 \mathrm{GHz}$ continuum TDM spectral window with DA59 as the reference antenna. Calibration solutions were calculated twice for phase and once for amplitude. The first phase solution interval was set to $300 \mathrm{~s}$, the second phase and amplitude solutions had the solution interval set equal to the binned integration time. Self-calibration solutions from the TDM spectral window were cross-applied to each FDM spectral window. Continuum subtraction of the line data was made in $u v$-space based on a single-order polynomial fit to all line-free channels in each spectral window. CLEAN imaging for the continuum was made using 


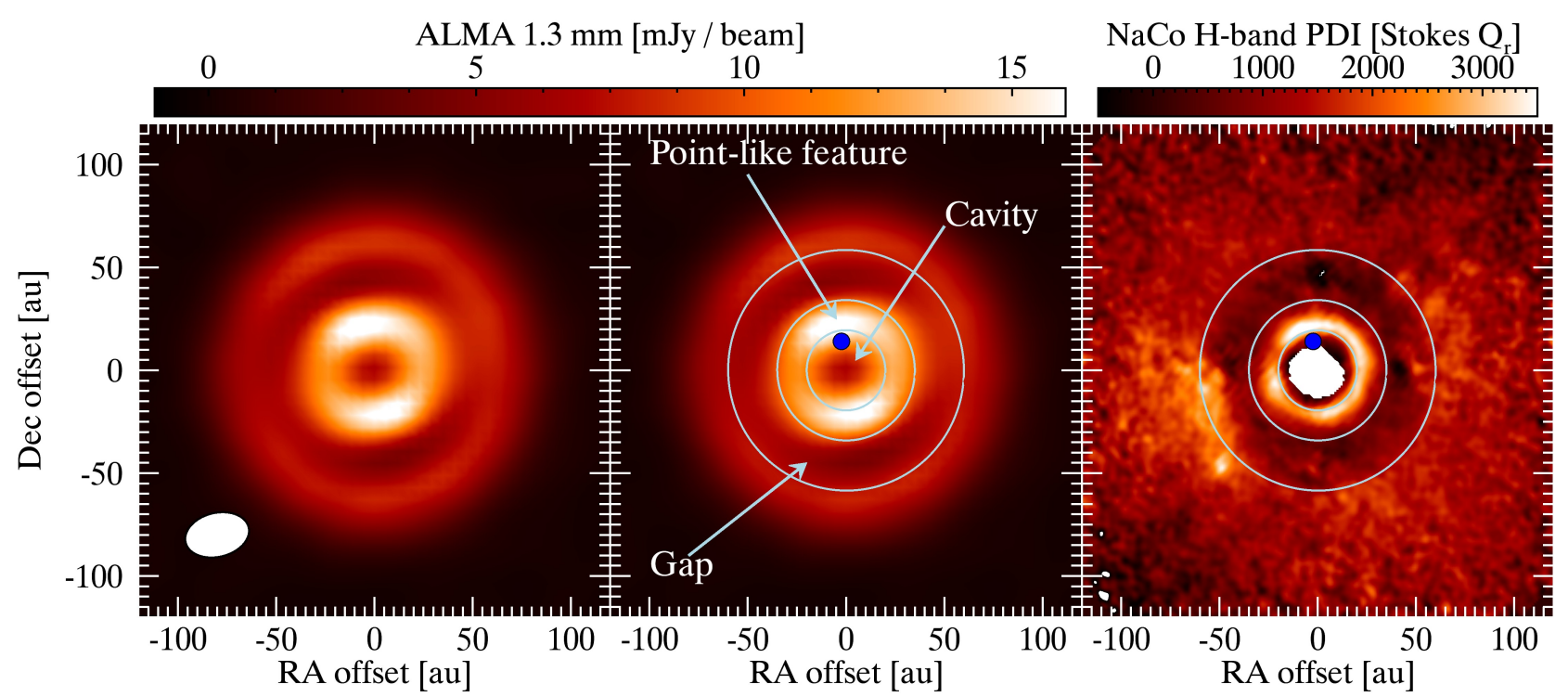

Fig. 1. (Left) ALMA $1.3 \mathrm{~mm}$ continuum map with Briggs weighting, robust $=0.5$. (Center) Overlaid with the position and size of the inner dust cavity and gap, and the position of the $L^{\prime}$-band point-like feature. (Right) NaCo H-band polarimetric differential image (Quanz et al. 2013).

different weighting schemes, Briggs (Briggs 1995), uniform and superuniform (Table 1). In the remaining paper we adopt the first (which provides the minimum rms). For the lines, CLEAN imaging was made with natural weighting. Data reduction was performed with the Common Astronomy Software Applications (CASA, McMullin et al. 2007).

\section{Results}

The $1.3 \mathrm{~mm}$ continuum and all the three CO isotopolog lines are readily detected. Figures $1-3$ show the dust continuum map, the line-integrated intensity maps, and the radial profiles, respectively. The channel maps of the three lines are presented in Figs. A.1-A.3 in the appendix.

\subsection{Dust continuum emission}

The $1.3 \mathrm{~mm}$ continuum map (Fig. 1) and the radial profile (Fig. 3) reveal a double-ring structure in the dust distribution with an inner cavity $\sim 0$ '. 17 in radius and a dust gap between $\sim 00^{\prime \prime} 28-0$ '.48, confirming the earlier findings of Osorio et al. (2014). The dust continuum emission drops steeply beyond $00^{\prime \prime} 64$. The different structures are highlighted in Fig. 1 (center panel) along with the position of the point-like $L^{\prime}$-band emission (Biller et al. 2014; Reggiani et al. 2014). The radial profile (Fig. 3) is shown at two different position angles, $\mathrm{PA}=5^{\circ}$ (major axis) and $\mathrm{PA}=95^{\circ}$ (minor axis). Along the minor axis, the continuum is slightly asymmetric, with the west side brighter than the east side. The flux difference between the two sides is $\sim 17 \mathrm{mJy}(\sim 2.5 \sigma)$.

The ALMA continuum map shows some similarities with the $H$-band polarimetric differential imaging (PDI, Quanz et al. 2013; Momose et al. 2015). The NaCo $H$-band PDI image is shown in Fig. 1, and Fig. 4 shows the radial intensity profile. The position and size of the inner dust ring and gap are consistent between both wavelength ranges. In the outer disk, the ALMA continuum emission is clearly more compact than the $H$-band emission (Fig. 4).

The dearth of dust continuum emission inside the inner dust cavity and the dust gap together with the similarities between the $H$-band PDI and the dust continuum emission suggest that the cavity and the gap are due to a substantial depletion of dust particles. An upper limit to the dust mass inside the gap can be estimated from the rms of the continuum flux density (Table 1). With the assumption of optically thin emission, the dust mass is (e.g., Roccatagliata et al. 2009)

$M_{\text {dust,gap }}=\frac{S_{v, \text { gap }} d^{2}}{k_{v} B_{v}\left(T_{\text {dust,gap }}\right)}$,

where $S_{v, \text { gap }}(\mathrm{Jy})$ is the upper limit on the flux density, $d(\mathrm{~cm})$ the distance, $k_{v}=2\left(\mathrm{~cm}^{2} \mathrm{~g}^{-1}\right)$ the mass absorption coefficient at $230 \mathrm{GHz}$ (Beckwith et al. 1990), $T_{\text {dust,gap }}(\mathrm{K})$ the dust temperature inside the gap and $B_{v}\left(\mathrm{Jy} \mathrm{sr}^{-1}\right)$ the Planck function. We assume $T_{\text {dust,gap }}=50 \mathrm{~K}$ (see Fig. 5). The flux density upper limit is computed by adopting a constant flux of $2.1 \times 10^{-4} \mathrm{Jy} \mathrm{beam}^{-1}$ (i.e., $3 \times \mathrm{rms}$ ) over the entire gap area. This corresponds to a dust mass $3 \sigma$ upper limit of $\sim 0.3 M_{\oplus}$. With the same assumptions, the dust mass upper limit inside the cavity (assuming $T_{\text {dust,cavity }}=150 \mathrm{~K}$, Fig. 5) is $\sim 10^{-2} M_{\oplus}$.

\subsection{CO isotopolog emission}

The integrated-intensity maps (Fig. 2) and the radial profile (Fig. 3) of the three $\mathrm{CO}$ isotopologs show different intensity distributions: the ${ }^{12} \mathrm{CO}$ emission is centrally concentrated with most of the line intensity originating within a $\sim 0^{\prime \prime}$.20 radius; the peak of the ${ }^{13} \mathrm{CO}$ emission corresponds to that of ${ }^{12} \mathrm{CO}$, but with a secondary ring-like structure farther out in the disk; in the case of $\mathrm{C}^{18} \mathrm{O}$, the emission map shows an inner (weak) ring centered on $\sim 0^{\prime \prime}$. $1-0^{\prime \prime} .2$ and a (strong) outer ring peaking at $\sim 0^{\prime \prime} .55$ with a tail up to $\sim 1^{\prime \prime}$.7. The gas emission is more extended than the dust continuum emission (Fig. 3). Moreover, the $H$-band scattered-light emission in the outer disk follows the same intensity distribution as that of ${ }^{12} \mathrm{CO}$.

The positions of the two $\mathrm{C}^{18} \mathrm{O}$ peaks are spatially coincident with the location of the dust rings. Along the disk minor axis, the $\mathrm{C}^{18} \mathrm{O}$ is slightly asymmetric, with the west side brighter than the east side (similar to the continuum asymmetry). The flux difference between the two peaks is $\sim 18 \mathrm{mJy}(\sim 3 \sigma)$. The line emission maps are consistent with a disk inclination of $13^{\circ}$ and a position angle of the disk major axis of $5^{\circ}$. 


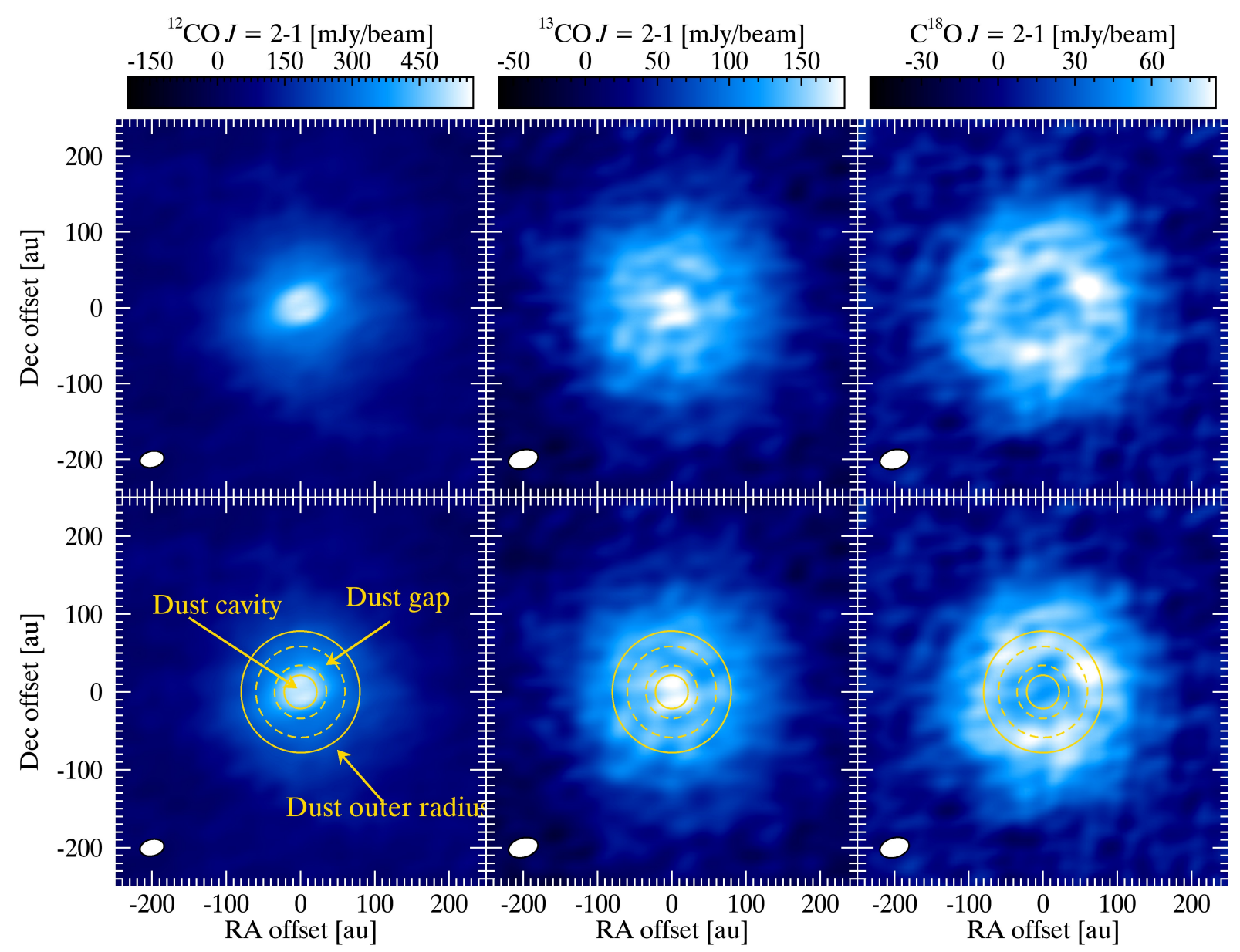

Fig. 2. (Top) Integrated intensity maps (natural weighting) of ${ }^{12} \mathrm{CO}($ left $),{ }^{13} \mathrm{CO}($ center $)$, and $\mathrm{C}^{18} \mathrm{O}($ right $) \mathrm{J}=2-1$, (bottom) overlaid with the dust rings structure.

The different radial distributions of the emission from the three isotopologs is readily explained by an optical depth effect because the $J=2-1$ transition of the three species has different $\tau$, with $\tau\left({ }^{12} \mathrm{CO}\right)>\tau\left({ }^{13} \mathrm{CO}\right)>\tau\left(\mathrm{C}^{18} \mathrm{O}\right)$. The optically thick ${ }^{12} \mathrm{CO}$ emission is mostly sensitive to the gas temperature, and as a consequence, its line intensity peaks toward the central hotter region of the disk. As the optical depth decreases, the line emission is less sensitive to the gas temperature and more sensitive to the gas column density. This is clear from the distribution of ${ }^{13} \mathrm{CO}$ and $\mathrm{C}^{18} \mathrm{O}$ : in the first case, the emission is less peaked (than ${ }^{12} \mathrm{CO}$ ) toward the central region, and it also shows a secondary peak (ring-shaped) in the outer colder disk. Finally, the optically thin $\mathrm{C}^{18} \mathrm{O}$ emission mostly originates in the outer disk, which shows a clear ring-like structure. The ring-like shape seen in the ${ }^{13} \mathrm{CO}$ and $\mathrm{C}^{18} \mathrm{O}$ emission map is spatially coincident with the outer dust ring.

\subsection{Disk surface density}

The spatial distribution of the emission of the three isotopologs provides direct insight into the gas content in the disk: the strong centrally peaked ${ }^{12} \mathrm{CO}$ emission indicates gas inside the dust gap and the dust cavity. On the other hand, the line intensity map of ${ }^{13} \mathrm{CO}$ and in particular $\mathrm{C}^{18} \mathrm{O}$ implies a substantial drop in gas surface density by a factor $\delta_{\text {gas }}$ on the order of $\sim 100$ inside the dust gap and cavity (see Sect. 5). The similar intensity profiles of the scattered light and ${ }^{12} \mathrm{CO}$ emission in the outer disk is a strong indication that the small dust grains are dynamically and thermally coupled to the gas in the outermost layers of the disk. The intensity drop in the inner disk is also clearly visible in the individual channel maps shown in Figs. A.1-A.3 in the appendix

The significance of the asymmetric emission along the minor axis (continuum and $\mathrm{C}^{18} \mathrm{O}$ ) is low $(\lesssim 3 \sigma)$, and more observations are needed to confirm this structure.

\section{Analysis}

In this section the ALMA observations of the $1.3 \mathrm{~mm}$ continuum and of the three $\mathrm{CO}$ isotopologs are compared with thermochemical disk model simulations. The goal is to quantify the decrease in dust and gas in the cavity and gap that we identified in the images.

\subsection{Disk model description}

The simulations presented here were generated using the thermo-chemical disk code DALI (Dust and Lines, Bruderer et al. 2012; Bruderer 2013). In this example, DALI takes as input a $T_{\text {eff }}=8400 \mathrm{~K}$ blackbody radiation field to simulate the stellar spectrum and a power-law surface density structure with an exponential tail

$\Sigma_{\mathrm{gas}}(R)=\Sigma_{\mathrm{c}}\left(\frac{R}{R_{\mathrm{c}}}\right)^{-\gamma} \exp \left[-\left(\frac{R}{R_{\mathrm{c}}}\right)^{2-\gamma}\right]$ 


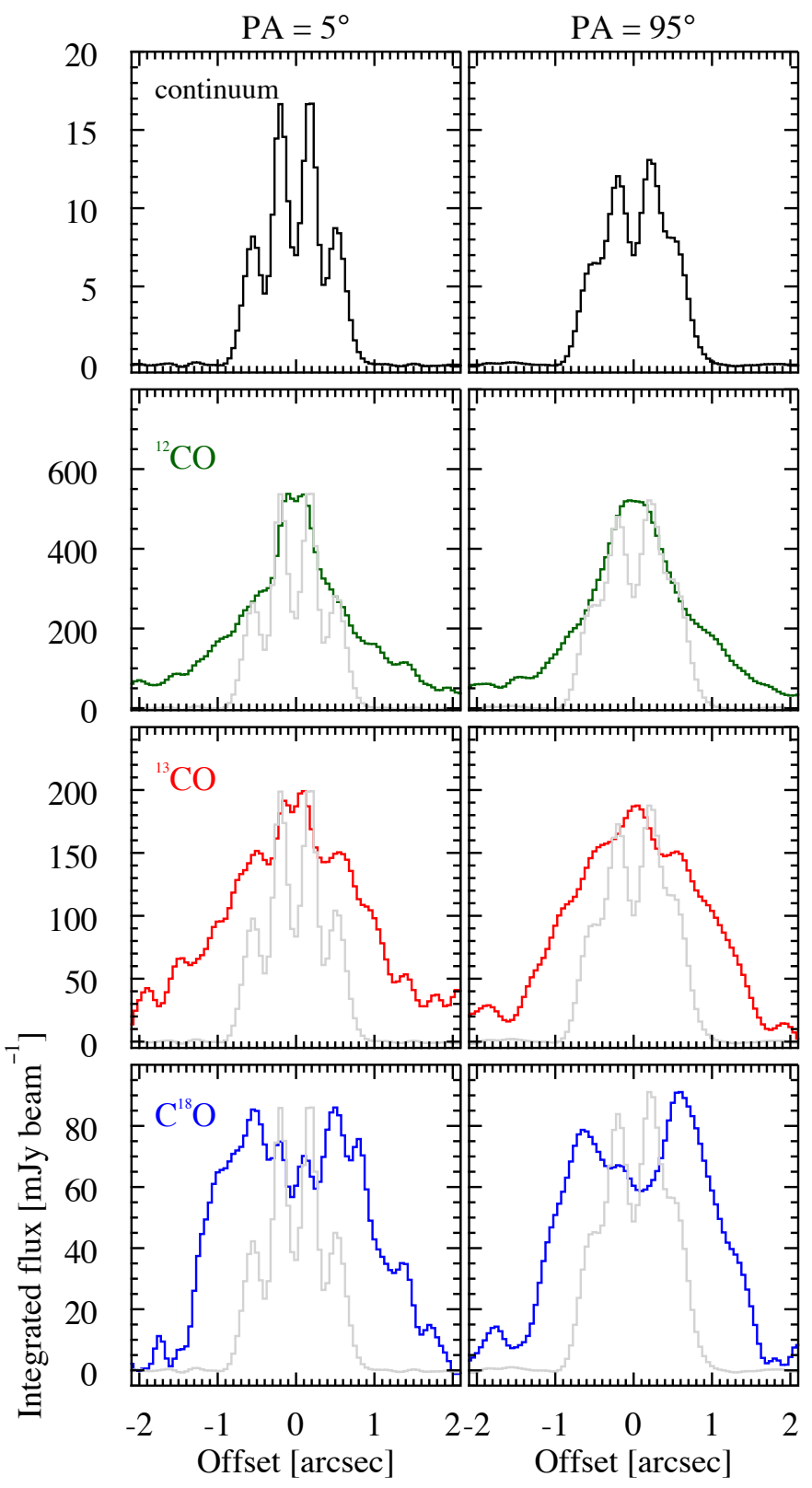

Fig. 3. Intensity profiles of the dust continuum and $\mathrm{CO}$ isotopolog integrated emission maps along the major $\left(\mathrm{PA}=5^{\circ}\right.$, left $)$ and minor $\left(\mathrm{PA}=95^{\circ}\right.$, right $)$ disk axis. The normalized continuum profile is overlaid (gray) on the $\mathrm{CO}$ panels for comparison. Note that the large-scale $\left(>2^{\prime \prime}\right)$ emission of ${ }^{12} \mathrm{CO}$ and ${ }^{13} \mathrm{CO}$ is the result of the reduced image quality (see Sect. 2).

with $R$ the radial distance from the star, $R_{\mathrm{c}}$ the critical radius, and $\Sigma_{\mathrm{c}}$ the gas surface density at $R=R_{\mathrm{c}}$. The dust surface density is $\Sigma_{\text {gas }} / \Delta_{\text {gd }}$, where $\Delta_{\text {gd }}$ is the gas-to-dust mass ratio. Along the vertical axis the gas density is parametrized by a Gaussian distribution with scale height $h(=H / R)$

$h=h_{\mathrm{c}}\left(\frac{R}{R_{\mathrm{c}}}\right)^{\psi}$,

with $h_{\mathrm{c}}$ the critical scale height and $\psi$ the flaring angle. Following D'Alessio et al. (2006), the settling of the large dust particles is implemented by adopting two different power-law grain size populations, small $(0.005-1 \mu \mathrm{m})$ and large $(0.005-1000 \mu \mathrm{m})$, and a power-law exponent $p=3.5$ (note that the size ranges

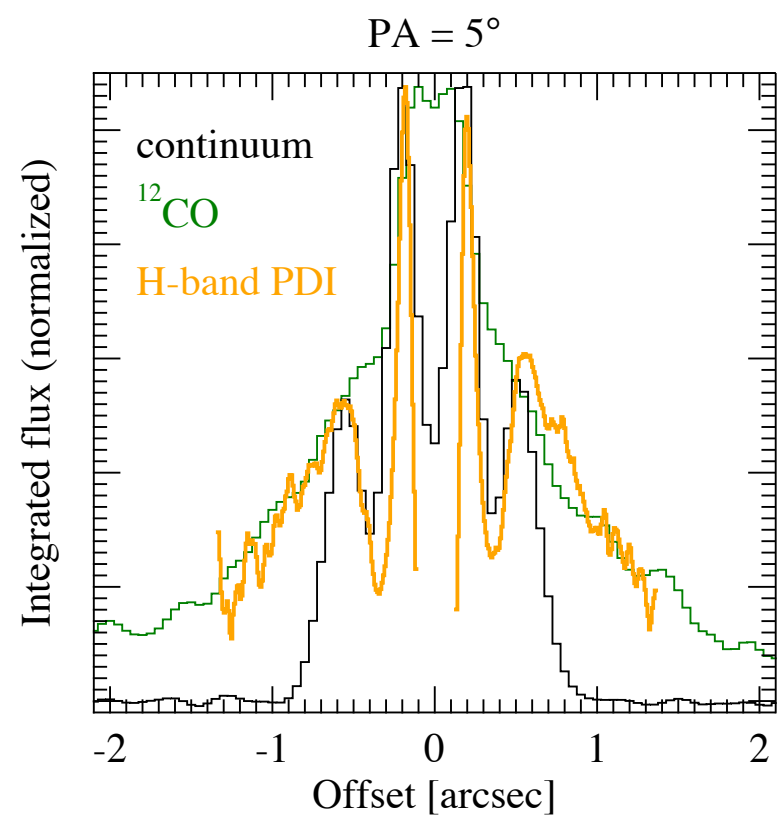

Fig. 4. Intensity profile of the $H$-band scattered light emission (azimuthally averaged to increase the signal-to-noise ratio) overlaid on the $1.3 \mathrm{~mm}$ continuum and ${ }^{12} \mathrm{CO}$ profiles.

are different from those adopted in D'Alessio et al. 2006). Dust mass absorption cross sections are adopted from Andrews et al. (2011). The scale height of the two populations is $h$ for the small grains and $\chi h(\chi<1)$ for the large grains. The mass ratio between the two populations is controlled by the parameter $f_{\text {large }}$ : the dust surface density is $\Sigma_{\text {dust }}\left(1-f_{\text {large }}\right)$ and $\Sigma_{\text {dust }} f_{\text {large }}$ for the small and large grains, respectively.

DALI solves the 2D dust continuum radiative transfer and determines the dust temperature and radiation field strength at each disk position. In a second step, DALI iteratively solves the gas thermal balance and chemistry, and returns as output the continuum and line emission maps computed through ray tracing. Isotope-selective photodissociation is included in the chemistry, as described in Miotello et al. (2014).

\subsection{Surface density}

Starting from the ALMA observations, we define a surface density structure as shown in Fig. 5 (left): the gas distribution extends from $R_{\text {gas in }}$ to $R_{\text {gas out }}$, while dust is only present between $R_{\text {dust in }}-R_{\text {gap in }}$ and between $R_{\text {gap out }}-R_{\text {dust out }}$. Along the radial axis, gas and dust densities are reduced by different decrease factors for the gas inside the dust cavity $\left(\delta_{\text {gas,cavity }}\right)$ and inside the dust gap $\left(\delta_{\text {gas,gap }}\right)$ and for the dust in the ring $\left(\delta_{\text {dust }}\right)$ as follows:

$n_{\text {gas }}= \begin{cases}0 & \text { for } R<R_{\text {gas in }} \\ n_{\text {gas }} \times \delta_{\text {gas,cavity }} & \text { for } R_{\text {gas in }}<R<R_{\text {dust in }} \\ n_{\text {gas }} \times \delta_{\text {gas,gap }} & \text { for } R_{\text {dust in }}<R<R_{\text {gap out }} \\ n_{\text {gas }} & \text { for } R_{\text {gap out }}<R<R_{\text {gas out }} \\ 0 & \text { for } R>R_{\text {gas out }}\end{cases}$

$n_{\text {dust }}= \begin{cases}0 & \text { for } R<R_{\text {dust in }} \\ n_{\text {dust }} \times \delta_{\text {dust }} & \text { for } R_{\text {dust in }}<R<R_{\text {gap in }} \\ 0 & \text { for } R_{\text {gap in }}<R<R_{\text {gap out }} \\ n_{\text {dust }} & \text { for } R_{\text {gap out }}<R<R_{\text {dust out }} \\ 0 & \text { for } R>R_{\text {dust out. }}\end{cases}$ 

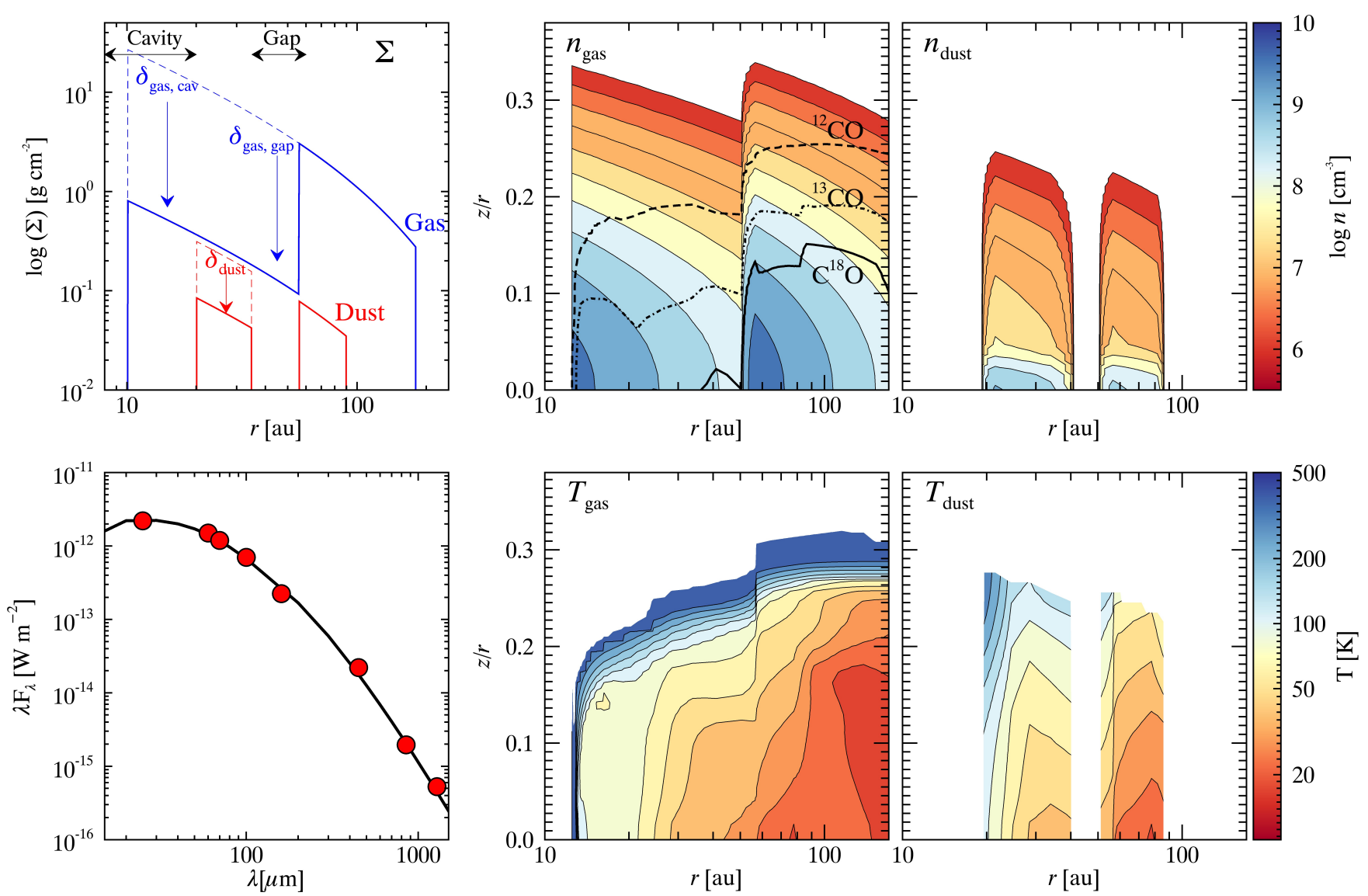

Fig. 5. Fiducial model: (top left) surface density profile; (top middle) gas density structure: the $\tau=1$ layer of the $J=2-1$ transition is overlaid as the dashed $\left({ }^{12} \mathrm{CO}\right)$, dot-dashed $\left({ }^{13} \mathrm{CO}\right)$, and solid $\left(\mathrm{C}^{18} \mathrm{O}\right)$ curve; (top right) dust density structure; (bottom left) spectral energy distribution with fiducial model (black solid curve): data (red dots) are from IRAS, Herschel (Pascual et al. 2016), SCUBA (Sandell et al. 2011), and ALMA (this work); (bottom middle) gas temperature structure; (bottom right) dust temperature structure.

Figure 5 shows the modeled disk density structure.

We note that our model does not include the small ring of hot dust inside 0.27 au (e.g., Wagner et al. 2015). This does not influence our results as the extinction produced by this small amount of dust is negligible and does not affect the propagation of the ultraviolet radiation farther out in the disk.

\subsection{Model grid}

We created a grid of disk models by varying the most relevant geometrical and physical parameters. These are

- the gas and dust mass (regulated by the combination of $\Sigma_{\mathrm{c}}$, $\gamma, R_{\mathrm{c}}$ and $\Delta_{\mathrm{gd}}$ ), which control the overall continuum and line emission as well as the SED;

- the flaring angle $(\psi)$, which mostly influences the radial intensity profile of the optically thick ${ }^{12} \mathrm{CO}$ emission (arising from the outermost layers);

- the scale height $\left(h_{\mathrm{c}}\right)$, which has a great effect on the intensity of the gas and dust emission and on the SED;

- the gas and dust decrease factors ( $\left.\delta_{\text {gas cavity }}, \delta_{\text {gas gap }}, \delta_{\text {dust }}\right)$, which control the gas and dust emission inside the dust gap;

- the size and position of the cavity and of the gap ( $R_{\text {gas in }}$, $\left.R_{\text {dust in }}, R_{\text {gap in }}, R_{\text {gap out }}\right)$; and

- the outer dust and gas radii ( $\left.R_{\text {dust out }}, R_{\text {gas out }}\right)$.

Table 2 lists the definition, range, and step size of the different parameters.
Using DALI, we created model images of the dust continuum emission and of the $J=2-1$ transition of the three $\mathrm{CO}$ isotopologs. In the case of the $\mathrm{CO}$ isotopolog lines, the model channel maps are computed with the same spectral resolution as the observations. From the DALI model images, we measured synthetic visibilities, synthetic observations, and residual (data-model) images reading the $u v$ coordinates, integration time, source position, hour angle, and spectral window parameters directly from the observed ALMA measurement set. For this task, we used the CASA tools SIMOBSERVE and CLEAN.

The model grid is compared to the observations with the aim of constructing a fiducial model that quantitatively reproduces the ALMA observations. The observed radial intensity profiles (along the disk minor and major axes) and the SED are fitted against the model grid. The fiducial model is defined by the set of parameters that minimize the difference between observations and model grid within the explored parameter space.

\subsection{Fiducial model and comparison with observations}

The surface density distribution, the density and temperature structure, and the SED of the fiducial model are shown in Fig. 5. Figures 6 and 7 show the comparison with the observations: Fig. 6 shows (from left to right) the ALMA observations (continuum or line-integrated map), synthetic observations, and residual images (computed in the uv-plane); Fig. 7 shows the radial intensity profiles along the disk major and minor axes. In order 
D. Fedele et al.: ALMA unveils rings and gaps in the protoplanetary system HD 169142: signatures of two giant protoplanets

Table 2. Fiducial disk model parameters.

\begin{tabular}{|c|c|c|c|}
\hline Parameter & \multicolumn{2}{|l|}{$\begin{array}{l}\text { Value } \\
\text { Fixed }\end{array}$} & Description \\
\hline & \multicolumn{2}{|l|}{ Fixed } & \\
\hline$M_{\star}\left[M_{\odot}\right]$ & \multicolumn{2}{|l|}{$1.65^{\diamond}$} & stellar mass \\
\hline$T_{\text {eff }}[\mathrm{K}]$ & \multicolumn{2}{|l|}{$8400^{\ddagger}$} & stellar temperature \\
\hline$L_{\star}\left[L_{\odot}\right]$ & \multicolumn{2}{|l|}{$10^{\dagger}$} & stellar luminosity \\
\hline$d[\mathrm{pc}]$ & \multicolumn{2}{|l|}{$117^{\dagger}$} & stellar distance \\
\hline$i\left[^{\circ}\right]$ & \multicolumn{2}{|l|}{$13^{\star, \dagger}$} & disk inclination \\
\hline $\mathrm{PA}\left[{ }^{\circ}\right]$ & \multicolumn{2}{|l|}{$5^{\star, \dagger}$} & disk position angle \\
\hline \multirow[t]{2}{*}{$\chi, f_{\text {large }}$} & \multirow{2}{*}{$\frac{0.2^{\dagger}}{\text { Range/step }}$} & & settling parameters \\
\hline & & Best fit & \\
\hline$\psi$ & $0.0-0.2 / 0.05$ & 0.0 & degree of flaring \\
\hline$\gamma$ & $0-2 / 0.5$ & 1.0 & $\Sigma(r)$ power-law exponent \\
\hline$h_{\mathrm{c}}$ & $0.05-0.09 / 0.01$ & 0.07 & scale height at $R=R_{\mathrm{c}}$ \\
\hline$R_{\mathrm{c}}[\mathrm{au}]$ & $50-200 / 50$ & 100 & critical radius \\
\hline$\Delta_{\mathrm{gd}}$ & $50-100 / 10$ & 80 & gas-to-dust mass ratio \\
\hline$\Sigma_{\mathrm{c}}\left[\mathrm{g} \mathrm{cm}^{-2}\right]$ & $5-8 / 0.5$ & 6.5 & $\Sigma_{\text {gas }}(R)$ at $R=R_{\mathrm{c}}$ \\
\hline$R_{\text {gas in }}[\mathrm{au}]$ & $11-15 / 1$ & 13 & gas inner radius \\
\hline$R_{\text {dust in }}[\mathrm{au}]$ & $18-22 / 1$ & 20 & dust inner radius \\
\hline$R_{\text {gap in }}[\mathrm{au}]$ & $32-36 / 1$ & 35 & dust cavity inner radius \\
\hline$R_{\text {gap out }}[\mathrm{au}]$ & $52-60 / 2$ & 56 & dust outer radius \\
\hline$R_{\text {gas out }}[\mathrm{au}]$ & $170-210 / 10$ & 180 & gas outer radius \\
\hline$R_{\text {dust out }}[\mathrm{au}]$ & $81-91 / 2$ & 83 & dust outer radius \\
\hline$\delta_{\text {dust }}$ & $0.25-0.35 / 0.02$ & 0.27 & dust depletion for $R_{\text {dust in }}<R<R_{\text {gap in }}$ \\
\hline$\delta_{\text {gas cavity }}$ & $0.01-0.05 / 0.005$ & 0.025 & gas depletion for $R_{\text {gas in }}<R<R_{\text {dust in }}$ \\
\hline$\delta_{\text {gas gap }}$ & $0.01-0.05 / 0.005$ & 0.025 & gas depletion for $R_{\text {dust in }}<R<R_{\text {gap in }}$ \\
\hline
\end{tabular}

References. ${ }^{(\diamond)}$ Blondel \& Djie (2006); ${ }^{(\star)}$ Dunkin et al. (1997); ${ }^{\star}$ Raman et al. (2006); ${ }^{(\dagger)}$ this work.

to asses the quality of the fit, Fig. B.1 shows the radial profile differences for a subset of the model grid.

Dust surface density: the fiducial model reproduces the dust continuum image well, with an inner dust cavity of $R \sim 20$ au, an inner ring between $\sim 20-35 \mathrm{au}$, a gap between $\sim 35-56 \mathrm{au}$, and an outer ring between $\sim 56-83$ au. The cavity and the gap are empty of millimeter-sized dust particles down to the noise level; there is an upper limit to the dust mass of $\sim 10^{-2} M_{\oplus}$ and $0.3 M_{\oplus}$ in the cavity and gap, respectively. The inner ring is decreased by a factor of $\sim 3.7\left(=1 / \delta_{\text {dust }}\right)$ (Fig. 3). The total dust mass is $1 \times 10^{-4} M_{\odot}$. The cavity and the gap are also free of micron-sized dust particles, as suggested by the NACO polarimetric observations (Fig. 4).

Gas in the inner disk: our analysis confirms the gas inside the dust gap (i.e., $35 \mathrm{au}<R<56$ au) and inside the dust cavity $(R<20 \mathrm{au})$ down to an inner radius of $\sim 13 \mathrm{au}$. The gas surface density inward of the dust gap is decreased by a factor of $\sim 30-40$ $\left(=1 / \delta_{\text {gas gap }}\right)$. Interestingly, we find that $\delta_{\text {gas cavity }}=\delta_{\text {gas gap }}$.

Gas in the outer disk: the gas surface density extends well beyond the dust outer radius. The fiducial model closely follows the slope of the radial profile of the three CO isotopologs in the outer disk. The outer gas radius of $180 \mathrm{au}$ is set by the steep drop of the $\mathrm{C}^{18} \mathrm{O}$ emission at large radii. Adopting a larger outer gas radius would overestimate the $\mathrm{C}^{18} \mathrm{O}$ emission in the outer disk, leaving the profiles of ${ }^{12} \mathrm{CO}$ and $\mathrm{C}^{13} \mathrm{O}$ almost unchanged. The total gas mass is $1.9 \times 10^{-2} M_{\odot}$ for the adopted standard carbon abundance of $[\mathrm{C}] /[\mathrm{H}]=2.4 \times 10^{-4}$. While the fiducial model reproduces the intensity profiles reasonably well, the absolute flux of the ${ }^{13} \mathrm{CO}$ emission is slightly underestimated. The difference in absolute flux is on the order of $\sim 10 \%$.

\section{Discussion}

The radial distribution of the (optically thin) $1.3 \mathrm{~mm}$ emission showing two deep gaps is qualitatively consistent with two giant planets shaping the dust distribution. Pinilla et al. (2015) performed 2D hydrodynamical simulations coupled with a dust evolution model of a disk hosting two giant planets, which showed that planets can produce multiple dust traps. These simulations show that the formation of dust traps is due to a combination of three main parameters: planetary mass, disk viscosity, and dust fragmentation velocity. In particular, the trap is easily formed for a high planetary mass and/or a low disk viscosity. In the case of HD 169142, the lack of dust continuum emission inside the dust cavity ( $R \lesssim 20 \mathrm{au}$ ) and in the outer gap (35 au $\lesssim \preccurlyeq 56 \mathrm{au}$ ) suggests very efficient dust trapping by means of two giant planets.

Azimuthal asymmetries are sometimes observed in the dust continuum emission of Herbig AeBe systems, as in the case of HD 142527 (Casassus et al. 2012; Fukagawa et al. 2013) and IRS 48 (van der Marel et al. 2013). Such an asymmetry is thought to be due to dynamical interaction between the disk and a massive planet generating a vortex where dust particles are trapped (Rossby instability, de Val-Borro et al. 2007; Lyra et al. 2009). The lack of such an asymmetry in the two dust rings of HD 169142 implies an upper limit to the two masses of the two planets of $\lesssim 10 M_{\text {Jupiter }}$.

The density drop of gas inward of the dust gap requires a rather massive planet with $M_{\text {planet }} \gtrsim 1 M_{\text {Jupiter }}$ for the outer planet. For a lower planetary mass, the cavity is "porous" and gas from the outer disk could flow inward and fill the gap (e.g., Lubow \& D'Angelo 2006; Alexander \& Armitage 2009). This is consistent with the prescriptions of Rosotti et al. (2016), which predict a mass of $\gtrsim 0.3 M_{\text {Jupiter }}$ (based on the size of the dust gap). 


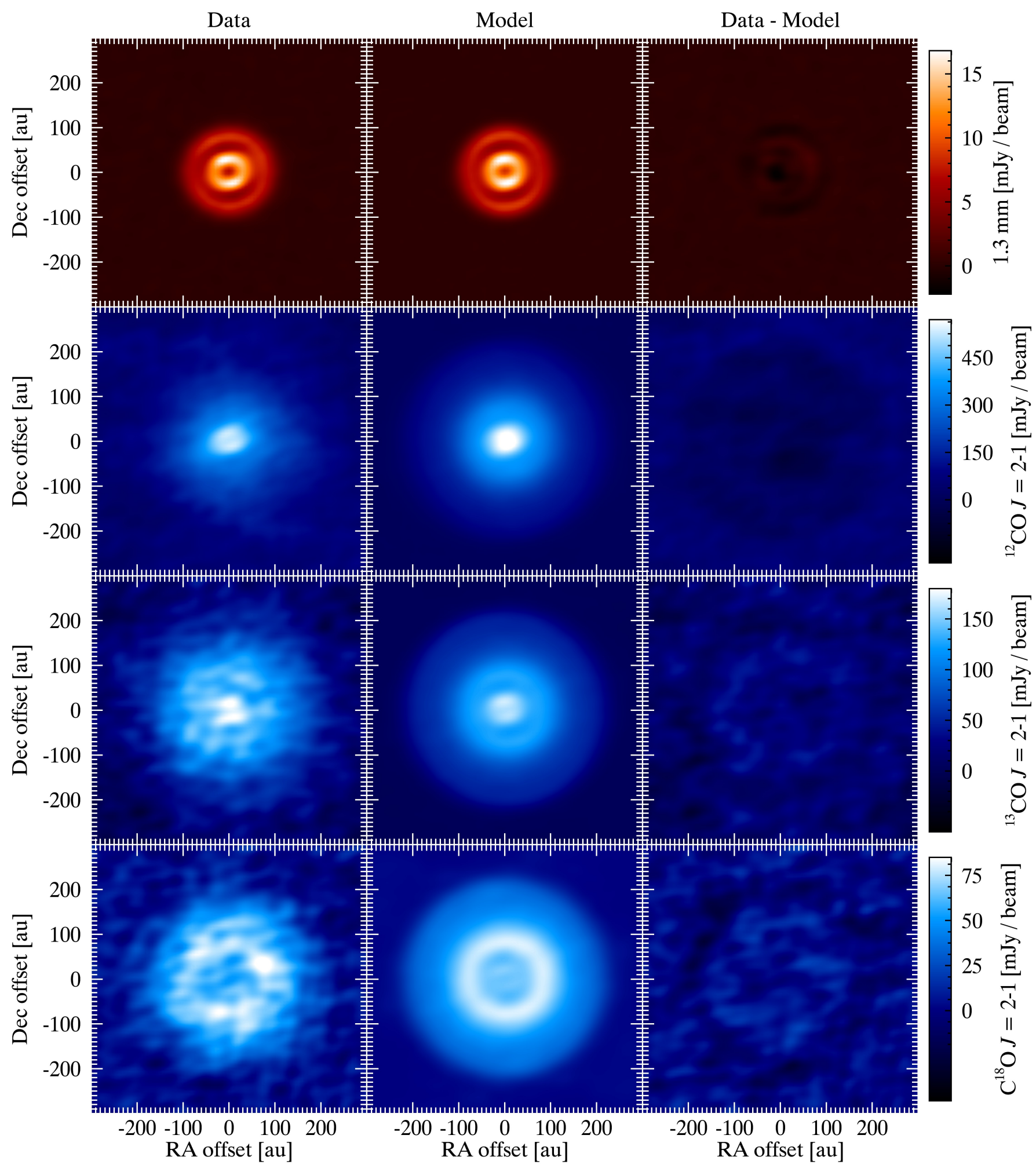

Fig. 6. Results of the fiducial model: (from left to right) ALMA image, model image, residual. Weighting scheme as in Figs. 1 and 2.

In the case of the inner dust cavity, the ALMA CO maps show no further drop of the gas density, suggesting inward gas flow. This poses a further limit to the planetary mass inside the inner dust cavity of $M_{\text {planet }}<M_{\text {Jupiter }}$.

Based on the lack of azimuthal asymmetric features and on the drop of the gas surface density, the ALMA data presented here are thus consistent with the presence of two giant planets of
$M_{\text {planet }} \sim 0.1-1 M_{\text {Jupiter }}$ and $M_{\text {planet }} \sim 1-10 M_{\text {Jupiter }}$ for the inner and outer planet, respectively.

The ALMA $1.3 \mathrm{~mm}$ continuum image confirms a real dust gap (i.e., depletion of dust particles) between $\sim 35-56$ au. This gap was previously detected through near-infrared polarimetric imaging, but only thanks to ALMA it is possible to determine the density drop. The dust gap is most likely the outcome 


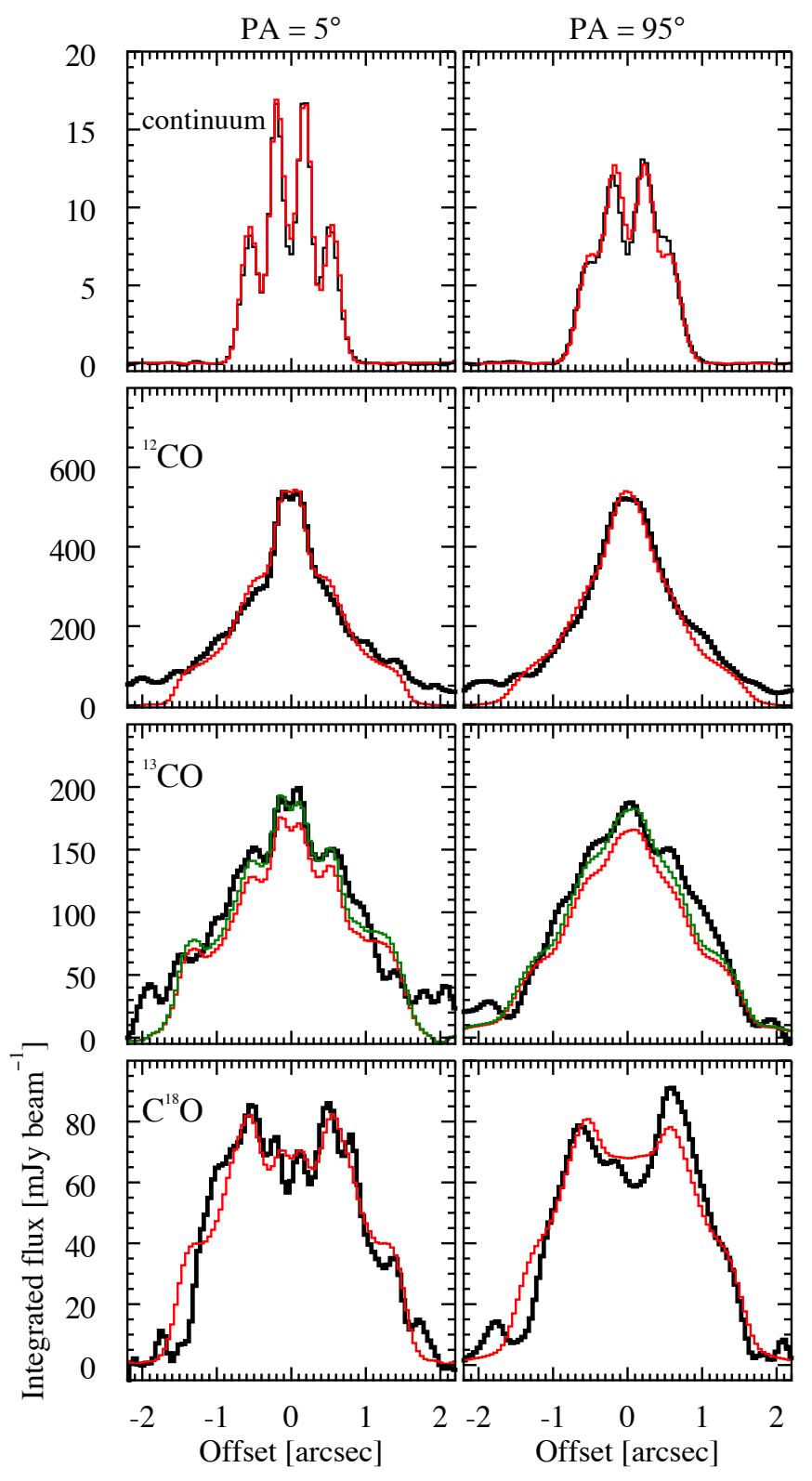

Fig. 7. Results of the fiducial model: comparison of the observed (black curve) and modeled (red curve) radial intensity profiles. In the case of ${ }^{13} \mathrm{CO}$, the green curve represents the fiducial model scaled up by $10 \%$ to match the absolute flux level.

of dynamical interaction between the disk and a second unseen planet, as noted above. Moreover, while the dust continuum emission is confined within a radius of $83 \mathrm{au}$, with a sharp decay, the gas emission extends up to $\sim 180$ au radius, as is shown in Figs. 2 and 3. This dichotomy is also observed in other systems that are observed at high angular resolution, such as LkCa 15 (Isella et al. 2012), HD 163296 (de Gregorio-Monsalvo et al. 2013), HD 100546 (Walsh et al. 2014), and HD 97048 (Walsh et al. 2016; van der Plas et al. 2017). The smaller size of the dust disk compared to the gas is primarily due to an optical depth effect (e.g., Dutrey et al. 1996), but the sharp drop in dust emission hints at the radial drift of the dust particles (Weidenschilling 1977; Birnstiel \& Andrews 2014), with the grains trapped at local pressure maxima induced by the planet-disk interaction. The similarity of the radial intensity profile of the $H$-band scattered light and of the
${ }^{12} \mathrm{CO}$ emission is indicative of dynamical and thermal coupling of the small dust grains with the gas in the outermost layers of the disk.

An alternative scenario that could also explain the surface density profile of HD 169142 is the case of magneto-rotational (MRI) instability creating dead-zones (e.g., Regály et al. 2012; Flock et al. 2015; Hasegawa \& Takeuchi 2015). While a deadzone itself results in a pressure bump with a gas density contrast of only a few, the combination with a mass-loss mechanism can cause this region to become largely devoid of gas by an amount similar to that observed in HD 169142. This process was investigated by Morishima (2012), for instance, in the case of MRI instability combined with photoevaporation, and by Pinilla et al. (2016) by including magnetohydrodynamics (MHD) wind (and dust evolution). However we note that no signature of mass loss is visible in the $\mathrm{CO}$ isotopolog channel maps.

Other protoplanetary systems around Herbig AeBe stars show similar multiple-ring dust distribution structures, such as HD 100546 (Walsh et al. 2014), HD 97048 (Walsh et al. 2016), and HD 163296 (Isella et al. 2016). In these cases, the ALMA observations also suggest several planets that are responsible for the dynamical clearing of the disk. The emerging picture is that these Herbig AeBe protoplanetary disks are in an late evolutionary phase where planets have already formed at large distances from the star. Future ALMA observations will tell us whether this is true for the entire class of Herbig AeBe systems.

\section{Conclusions}

From the analysis of the ALMA observations presented in this paper we conclude that gas and dust in HD 169142 are physically decoupled, with the dust particles concentrated in two rings between $\sim 20-35$ au and $\sim 56-83$ au. Thanks to ALMA, we have for the first time strong constraints on the distribution and dynamics of the bulk of the dust and gas. We find a real dust depletion (i.e., absence of dust particles) inside the cavity ( $R \lesssim 20 \mathrm{au}$ ) and the gap (35 $\lesssim \lesssim 56 \mathrm{au}$ ). The dust cavity and gap are filled in with gas, as suggested by the emission maps of the three $\mathrm{CO}$ isotopologs, with the gas surface density reduced by a factor of $\sim 30-40$ for $R \lesssim 56$ au. The sharp edge of the continuum map at 83 au is indicative of a radial drift of dust grains (dust outer radius $\ll$ gas outer radius).

Of the various theories proposed to explain the opening of gaps in disks (e.g., dynamical interaction with planets, MRI, dust sintering, and photoevaporation), the most likely scenario for HD 169142 is that several giant planets ( $\left.\gtrsim M_{\text {Jupiter }}\right)$ have carved out the disk and caused the cavity and the gap and trapped the dust particles beyond the planetary orbits. The combined effect of MRI instability (forming dead-zones) and MHD wind could also cause the ring and gap structure in both gas and dust, although with different characteristics, which may be tested by future data with higher signal-to-noise ratio and higher angular resolution.

We stress the importance of spatially resolved observations of multiple $\mathrm{CO}$ isotopolog transitions: thanks to the different optical depths, the spatially resolved channel maps of the three isotopologs allowed us to detect and quantify the very small amount $\left(\lessgtr 0.1 M_{\text {Jupiter }}\right)$ of gas inside the dust gap. This demonstrates the potential of $\mathrm{CO}$ isotopolog observations in probing the gas surface density and the evolutionary phase of protoplanetary systems.

Acknowledgements. This paper makes use of the following ALMA data: ADS/JAO.ALMA\#2013.1.00592.S. ALMA is a partnership of ESO (representing its member states), NSF (USA) and NINS (Japan), together with NRC 
(Canada), NSC and ASIAA (Taiwan), and KASI (Republic of Korea), in cooperation with the Republic of Chile. The Joint ALMA Observatory is operated by ESO, AUI/NRAO and NAOJ. This work has made use of data from the European Space Agency (ESA) mission Gaia (http://www. cosmos.esa.int/ gaia), processed by the Gaia Data Processing and Analysis Consortium (DPAC, http://www. cosmos.esa.int/web/gaia/dpac/consortium). Funding for the DPAC has been provided by national institutions, in particular the institutions participating in the Gaia Multilateral Agreement. D.F. acknowledges support from the Italian Ministry of Education, Universities and Research, project SIR (RBSI14ZRHR). M.C. and M.R.H. are supported by a TOP grant from the Netherlands Organisation for Scientific Research (NWO, 614.001.352). C.W. acknowledges financial support from the Netherlands Organisation for Scientific Research (NWO, grant 639.041.335) and start-up funds from the University of Leeds, UK. The authors thank M. Tazzari, S. Facchini, G. Rosotti, L. Testi, and P. Pinilla for useful discussions and S. Quanz for providing the NaCo image.

\section{References}

Alexander, R. D., \& Armitage, P. J. 2009, ApJ, 704, 989

ALMA Partnership, Brogan, C. L., Pérez, L. M., et al. 2015, ApJ, 808, L3

Andrews, S. M., Wilner, D. J., Espaillat, C., et al. 2011, ApJ, 732, 42

Andrews, S. M., Wilner, D. J., Zhu, Z., et al. 2016, ApJ, 820, L40

Beckwith, S. V. W., Sargent, A. I., Chini, R. S., \& Guesten, R. 1990, AJ, 99, 924

Biller, B. A., Males, J., Rodigas, T., et al. 2014, ApJ, 792, L22

Birnstiel, T., \& Andrews, S. M. 2014, ApJ, 780, 153

Blondel, P. F. C., \& Djie, H. R. E. T. A. 2006, A\&A, 456, 1045

Briggs, D. S. 1995, Ph.D. Thesis, The New Mexico Institute of Mining and Technology, Socorro, New Mexico, USA

Bruderer, S. 2013, A\&A, 559, A46

Bruderer, S., van Dishoeck, E. F., Doty, S. D., \& Herczeg, G. J. 2012, A\&A, 541, A91

Casassus, S., Perez M., S., Jordán, A., et al. 2012, ApJ, 754, L31

D’Alessio, P., Calvet, N., Hartmann, L., Franco-Hernández, R., \& Servín, H. 2006, ApJ, 638, 314

de Gregorio-Monsalvo, I., Ménard, F., Dent, W., et al. 2013, A\&A, 557, A133

de Val-Borro, M., Artymowicz, P., D’Angelo, G., \& Peplinski, A. 2007, A\&A, 471,1043

de Zeeuw, P. T., Hoogerwerf, R., de Bruijne, J. H. J., Brown, A. G. A., \& Blaauw, A. $1999, \mathrm{AJ}, 117,354$

Dunkin, S. K., Barlow, M. J., \& Ryan, S. G. 1997, MNRAS, 286, 604

Dutrey, A., Guilloteau, S., Duvert, G., et al. 1996, A\&A, 309, 493

Ercolano, B., Rosotti, G. P., Picogna, G., \& Testi, L. 2017, MNRAS, 464, L95

Fedele, D., Bruderer, S., van Dishoeck, E. F., et al. 2013, A\&A, 559, A77

Flock, M., Ruge, J. P., Dzyurkevich, N., et al. 2015, A\&A, 574, A68

Fukagawa, M., Tamura, M., Itoh, Y., et al. 2010, PASJ, 62, 347
Fukagawa, M., Tsukagoshi, T., Momose, M., et al. 2013, PASJ, 65, L14 Gaia Collaboration (Brown, A. G. A., et al.) 2016, A\&A, 595, A2 Grady, C. A., Schneider, G., Hamaguchi, K., et al. 2007, ApJ, 665, 1391 Hasegawa, Y., \& Takeuchi, T. 2015, ApJ, 815, 99

Honda, M., Maaskant, K., Okamoto, Y. K., et al. 2012, ApJ, 752, 143

Isella, A., Pérez, L. M., \& Carpenter, J. M. 2012, ApJ, 747, 136 Isella, A., Guidi, G., Testi, L., et al. 2016, Phys. Rev. Lett., 117, 251101 Lubow, S. H., \& D'Angelo, G. 2006, ApJ, 641, 526

Lyra, W., Johansen, A., Klahr, H., \& Piskunov, N. 2009, A\&A, 493, 1125

Malfait, K., Bogaert, E., \& Waelkens, C. 1998, A\&A, 331, 211

McMullin, J. P., Waters, B., Schiebel, D., Young, W., \& Golap, K. 2007, in Astronomical Data Analysis Software and Systems XVI, eds. R. A. Shaw, F. Hill, \& D. J. Bell, ASP Conf. Ser., 376, 127

Meeus, G., Pinte, C., Woitke, P., et al. 2010, A\&A, 518, L124

Miotello, A., Bruderer, S., \& van Dishoeck, E. F. 2014, A\&A, 572, A96

Momose, M., Morita, A., Fukagawa, M., et al. 2015, PASJ, 67, 83

Morishima, R. 2012, MNRAS, 420, 2851

Okuzumi, S., Momose, M., Sirono, S.-I., Kobayashi, H., \& Tanaka, H. 2016, ApJ, 821, 82

Osorio, M., Anglada, G., Carrasco-González, C., et al. 2014, ApJ, 791, L36

Panić, O., Hogerheijde, M. R., Wilner, D., \& Qi, C. 2008, A\&A, 491, 219

Papaloizou, J., \& Lin, D. N. C. 1984, ApJ, 285, 818

Pascual, N., Montesinos, B., Meeus, G., et al. 2016, A\&A, 586, A6

Pinilla, P., de Juan Ovelar, M., Ataiee, S., et al. 2015, A\&A, 573, A9

Pinilla, P., Flock, M., Ovelar, M. d. J., \& Birnstiel, T. 2016, A\&A, 596, A81

Pinte, C., Woitke, P., Ménard, F., et al. 2010, A\&A, 518, L126

Quanz, S. P., Avenhaus, H., Buenzli, E., et al. 2013, ApJ, 766, L2

Raman, A., Lisanti, M., Wilner, D. J., Qi, C., \& Hogerheijde, M. 2006, AJ, 131, 2290

Regály, Z., Juhász, A., Sándor, Z., \& Dullemond, C. P. 2012, MNRAS, 419, 1701

Reggiani, M., Quanz, S. P., Meyer, M. R., et al. 2014, ApJ, 792, L23

Roccatagliata, V., Henning, T., Wolf, S., et al. 2009, A\&A, 497, 409

Rosotti, G. P., Juhasz, A., Booth, R. A., \& Clarke, C. J. 2016, MNRAS, 459, 2790

Sandell, G., Weintraub, D. A., \& Hamidouche, M. 2011, ApJ, 727, 26

The, P. S., de Winter, D., \& Perez, M. R. 1994, A\&AS, 104, 315

van der Marel, N., van Dishoeck, E. F., Bruderer, S., et al. 2013, Science, 340, 1199

van der Marel, N., van Dishoeck, E. F., Bruderer, S., et al. 2016, A\&A, 585, A58 van der Plas, G., Wright, C. M., Ménard, F., et al. 2017, A\&A, 597, A32

Wagner, K. R., Sitko, M. L., Grady, C. A., et al. 2015, ApJ, 798, 94

Walsh, C., Juhász, A., Pinilla, P., et al. 2014, ApJ, 791, L6

Walsh, C., Juhász, A., Meeus, G., et al. 2016, ApJ, 831, 200

Weidenschilling, S. J. 1977, MNRAS, 180, 57

Zhang, K., Blake, G. A., \& Bergin, E. A. 2015, ApJ, 806, L7 
D. Fedele et al.: ALMA unveils rings and gaps in the protoplanetary system HD 169142: signatures of two giant protoplanets

\section{Appendix A: Channel maps}

The individual channel maps of ${ }^{12} \mathrm{CO},{ }^{13} \mathrm{CO}$, and $\mathrm{C}^{18} \mathrm{O}$ are shown in Figs. A.1, A.2, and A.3, respectively. The velocity scale is defined in the local standard of rest (LSR) system. In all cases, the maps are created using natural weighting, and the velocity resolution is resampled to $0.160 \mathrm{~km} \mathrm{~s}^{-1}\left({ }^{12} \mathrm{CO}\right)$ and $0.168 \mathrm{~km} \mathrm{~s}^{-1}$ $\left({ }^{13} \mathrm{CO}\right.$ and $\left.\mathrm{C}^{18} \mathrm{O}\right)$ with Hanning smoothing. The disk emission is detected from $v_{\mathrm{LSR}}=4.9 \mathrm{~km} \mathrm{~s}^{-1}$ to $v_{\mathrm{LSR}}=8.6 \mathrm{~km} \mathrm{~s}^{-1}$, and the systemic velocity is $v_{\mathrm{LSR}}=6.9 \mathrm{~km} \mathrm{~s}^{-1}$.

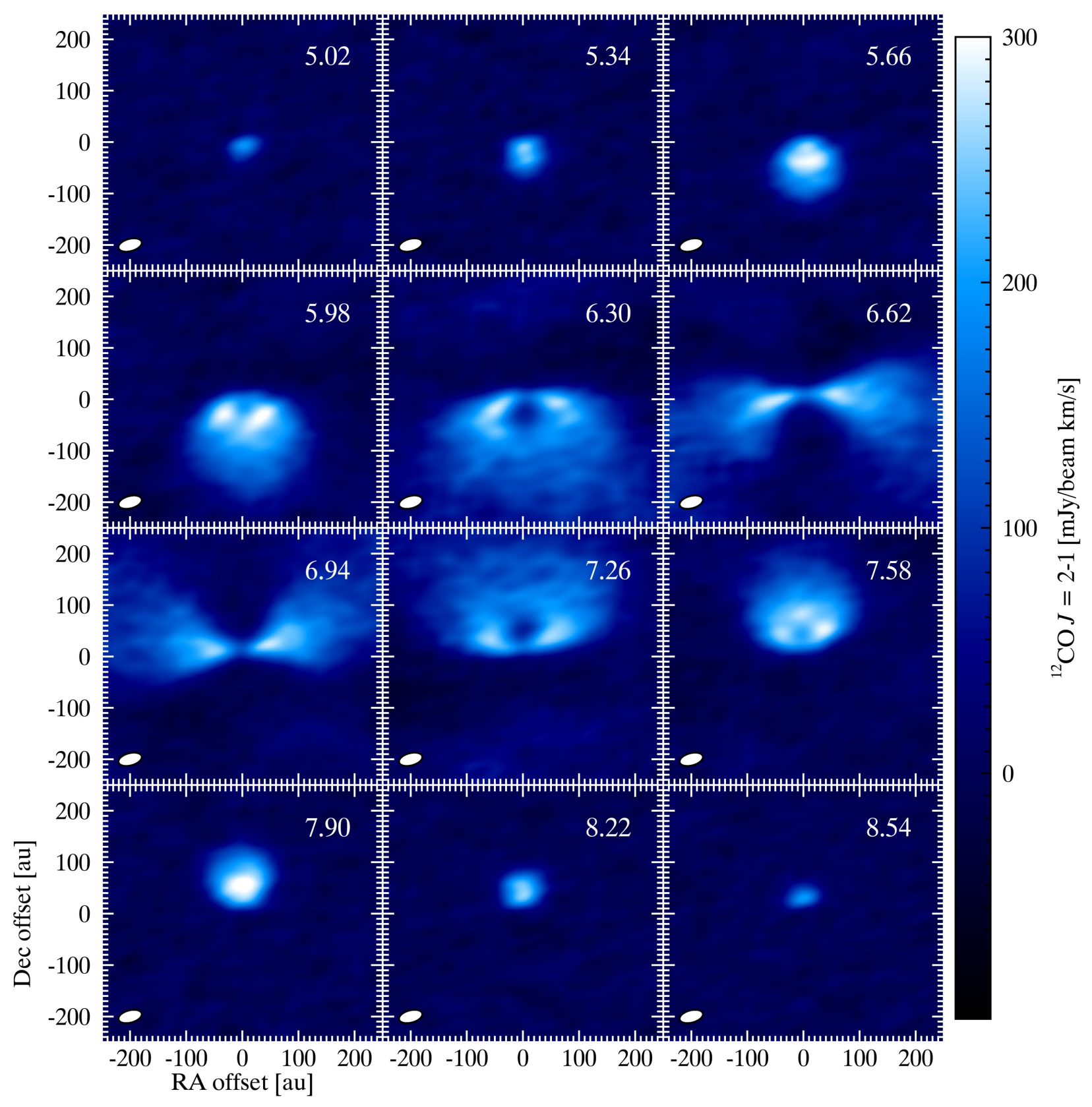

Fig. A.1. ALMA channel maps of ${ }^{12} \mathrm{CO} J=2-1$. 


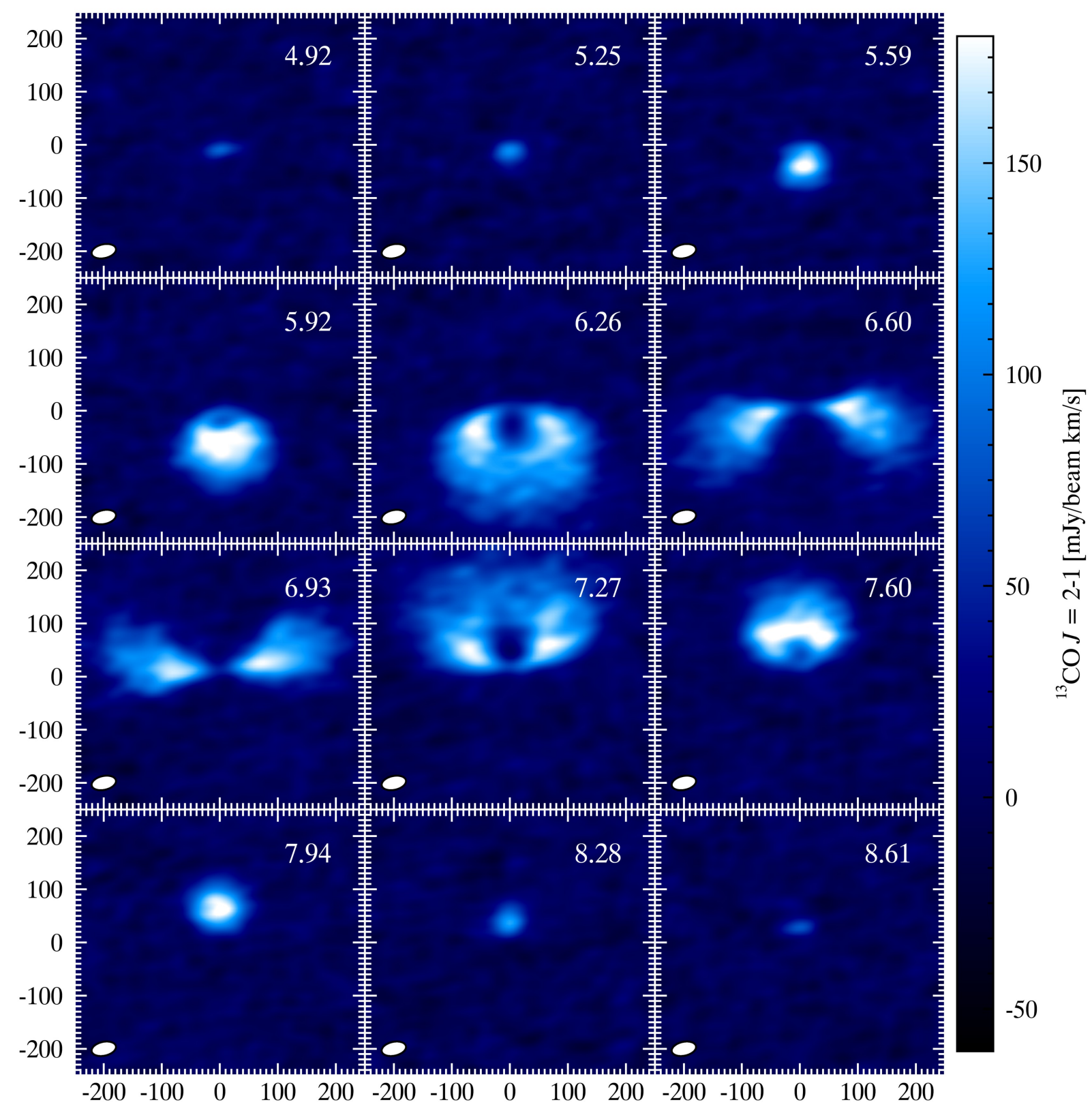

Fig. A.2. ALMA channel maps of ${ }^{13} \mathrm{CO} J=2-1$. 
D. Fedele et al.: ALMA unveils rings and gaps in the protoplanetary system HD 169142: signatures of two giant protoplanets

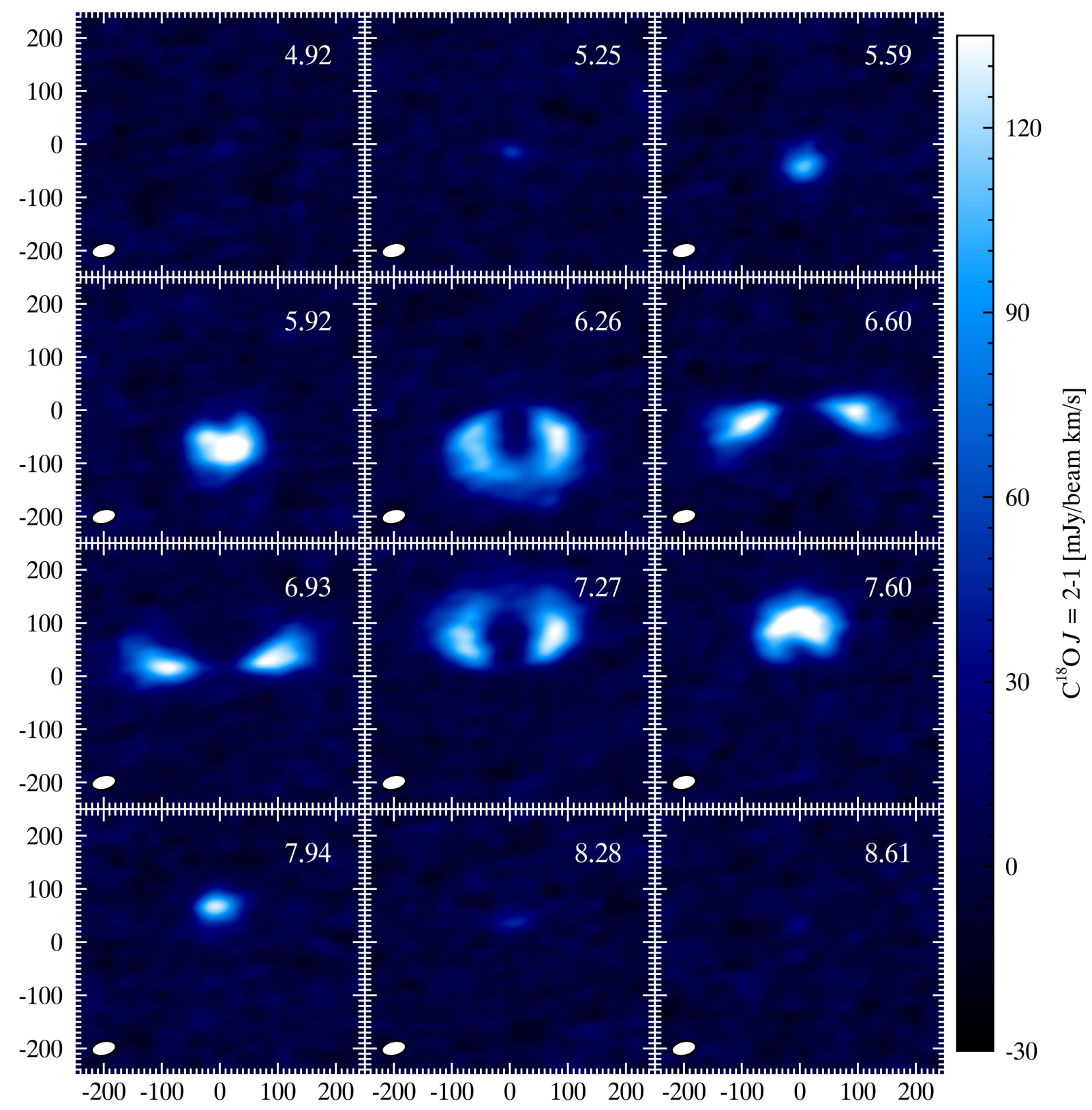

Fig. A.3. ALMA channel maps of $\mathrm{C}^{18} \mathrm{O} J=2-1$. 


\section{Appendix B: Model grid}

Figure B.1 shows the results of the model grid (Sect. 5.3) where we vary one parameter at a time. Only a subset of the model grid is shown here. We note in particular that the ${ }^{12} \mathrm{CO}$ radial profile is very sensitive to $R_{\text {gas in }}$ (the inner gas radius), while the inner dust radius $\left(R_{\text {cav }}\right)$ affects not only the continuum radial profile, but also the strength of the ${ }^{13} \mathrm{CO}$ and $\mathrm{C}^{18} \mathrm{O}$ emission in the inner disk.

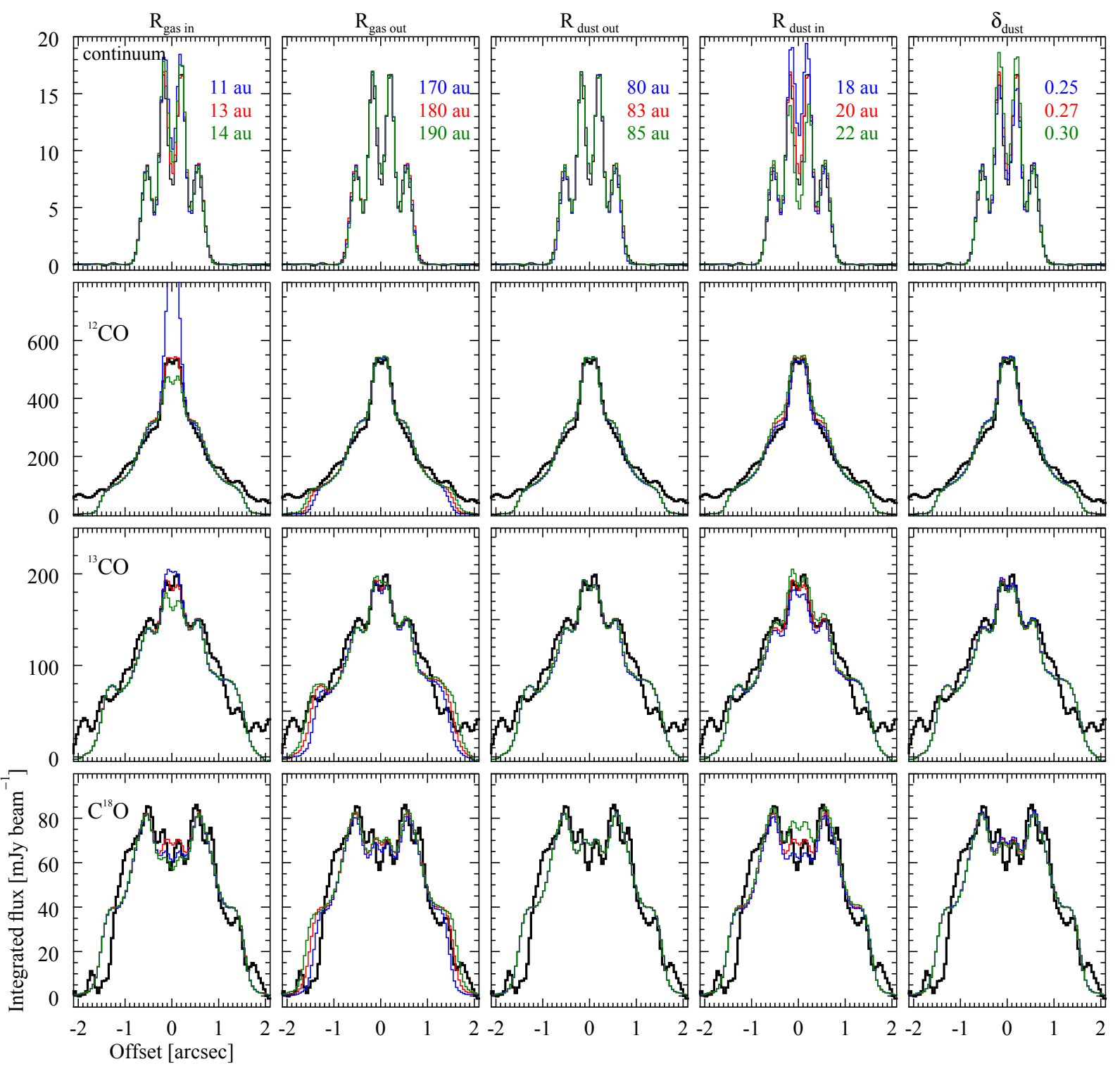

Fig. B.1. Radial intensity profile differences $\left(\mathrm{PA}=5^{\circ}\right)$ for a subset of the model grid. The ${ }^{13} \mathrm{CO}$ models are scaled up by $10 \%$ to match the absolute flux level of the observations. 